\title{
Factores asociados al estrés laboral en policías penitenciarios costarricenses
}

\section{Factors Related to Workplace Stress among Costa Rican Correctional Officers}

\author{
Tatiana María Blanco-Álvarez, MSc., \\ Megan A. Thoen, Ph.D. \\ Texas Tech University, Estados Unidos de América
}

\begin{abstract}
Resumen
El objetivo de esta investigación fue determinar si una muestra de policías penitenciarios, en Costa Rica, experimenta estrés laboral y si está relacionado con la presencia de estrés, depresión, ansiedad, burnout y otras características personales y ocupacionales. Además, se determina si hay diferencias entre hombres y mujeres con respecto a la presencia de tales factores. La Escala de Depresión, Ansiedad y Estrés (DASS), la Escala de Estrés Laboral para Oficiales Correccionales (EELOC), y el Cuestionario Breve de Burnout (CBB) fueron administrados a 66 oficiales correccionales de diferentes prisiones en Costa Rica. Conclusiones: El estrés laboral está significativamente relacionado con la presencia de estrés, ansiedad y depresión, pero no relacionado con la presencia de burnout. No se encontraron diferencias entre hombres y mujeres en ninguna variable. Es importante saber cómo el ambiente de trabajo afecta las vidas de los oficiales con el fin de mejorar las condiciones de trabajo que podrían tener efectos negativos sobre el desempeño en el trabajo y la salud, así como proporcionar información para los psicólogos policiales, que están a cargo de proveer servicios clínicos y de consejería a los oficiales correccionales.
\end{abstract}

Palabras clave: oficiales correccionales, estrés laboral, estrés, ansiedad, burnout, depresión

Tatiana María Blanco-Álvarez y Megan A. Thoen; Texas Tech University, Estados Unidos de América.

La correspondencia en relación con este artículo se dirige a Tatiana María Blanco-Álvarez,Programa de Análisis y Resolución de Conflictos, Nova Southeastern University en Florida, Estados Unidos, E-mail: tb1573@mynsu.nova.edu 


\begin{abstract}
The aim of this study was to establish whether a sample of correctional officers in Costa Rica experience workplace stress and if workplace stress is related to the presence of stress, depression, anxiety, burnout and other various personal and occupational characteristics. Additionally, whether there are differences between men and women regarding the presence of such factors are explored. The Depression, Anxiety and Stress Scale (DASS), Work Stress Scale for Correctional Officers (WSSCO) and Brief Questionnaire on Burnout (CBB) were administered to 66 correctional officers from different prisons in Costa Rica. Conclusions: Workplace stress is significantly related to the presence of stress, anxiety and depression, but not related to the presence of burnout. No differences between men and women were found on any variable. It is important to know how the work environment affects the lives of the officers, in order to improve the working conditions that could have negative effects on the job performance and health of the officers, as well to provide information for police psychologists who are in charge of providing clinical and counseling services to correctional officers.
\end{abstract}

Keywords: Correctional Officers, Workplace Stress, Stress, Anxiety, Burnout, Depression

Según el Reglamento General de la Policía Penitenciaria (1997), los policías penitenciarios son los encargados de controlar y supervisar todas las prisiones en el país. Además, los agentes están a cargo de la custodia, la vigilancia y el mantenimiento del orden y la disciplina en las cárceles, y de prestar atención a las personas privadas de libertad 24 horas, siete días a la semana (Reglamento General de la Policía Penitenciaria, 1997). Debido a esto, los policías están expuestos a epidemias, disturbios, huelgas de hambre, peleas, intentos de suicidio e intentos de asesinato por parte de las personas privadas de libertad (PPL). La exposición a estas situaciones puede dar lugar a la presencia de estrés laboral, estrés, ansiedad y depresión (Blanco \& Mora, 2015).

Desde un punto de vista médico y de la salud, el estrés se define como “(...) un estímulo físico o psicológico que puede producir tensión mental o reacciones fisiológicas que pueden conducir a la enfermedad" (Chandra \& Parvez, 2016, p.456). También, se puede conceptualizar como "(...) un estado desagradable de activación emocional y fisiológica que experimentan las personas en situaciones que ellos perciben como peligrosas o que amenazan de su bienestar" (Tenibiaje, 2013, p.74). Los estresores pueden clasificarse en tres categorías: a) los eventos catastróficos, b) los principales cargos de la vida y c) los problemas cotidianos; en el caso del estrés laboral, se incluyen exigencias cuantitativas (por ejemplo, la presión del tiempo), demandas cognitivas (como la dificultad del trabajo) y las demandas emocionales (como la empatía requerida) (Tenibiaje, 2013). Todo ello se ha relacionado con el aumento en el consumo de drogas, especialmente el tabaco y las deficiencias del sistema inmunitario (Campbell \& Henderson, 2016). Cuando el estrés se presenta en el contexto laboral, estamos hablando de estrés laboral o estrés relacionado con el trabajo.

De acuerdo con la Organización Mundial de la Salud (OMS, 2016), el estrés laboral es la respuesta fisiológica que se presenta cuando las exigencias y las presiones del trabajo no coinciden con los conocimientos y habilidades de las personas, y esto pone en riesgo su capacidad para hacer frente a dicha 
situación. Puede ser causado por el trabajo en sí, o por discrepancias entre las exigencias del trabajo y la personalidad de los trabajadores (Campbell \& Henderson, 2016), o por la mala organización del trabajo, el mal diseño de los puestos de trabajo, la mala administración y la falta de apoyo de la sede (OMS, 2016). Es importante tener en cuenta que el estrés laboral es un concepto diferente del burnout. Según Maslach y Jackson (1981), el burnout es un síndrome de agotamiento emocional y cinismo que ocurre a menudo entre las personas que trabajan en el cuidado de otros seres humanos. La principal diferencia entre ambos consiste en que el estrés laboral se trata de una respuesta fisiológica, mientras tanto el burnout es un síndrome, con diferentes síntomas, que caracterizan una enfermedad o una patología.

Según Finney, Stergiopoulos, Hensel, Bonito y Dewa (2013), existen cuatro categorías de estrés laboral: 1) estresores intrínsecos al trabajo: los que causan sobrecarga de trabajo y dificultad; 2) factores estresantes relacionados con el rol dentro de la organización: no tener autoridad para mejorar las condiciones que facilitan la actuación profesional; 3) estresores del desarrollo profesional: aquellos que afectan el futuro del trabajador en la organización y 4) factores estresantes de las relaciones laborales: interacciones estresantes entre los oficiales penitenciarios y sus supervisores o con las PPL. En el caso de los policías penitenciarios, el estrés laboral se ha asociado con la presencia de enfermedades como la hipertensión, las enfermedades del corazón, la depresión y la ansiedad (Misis, Kim, Cheeseman, Hogan, \& Lambert, 2013). Otros factores y fuentes de estrés laboral específicos de los establecimientos penitenciarios incluyen a) sobrecarga de trabajo, b) falta de autoeficacia, c) seguridad del trabajo, d) relaciones con otros compañeros de trabajo, e) problemas con las PPL, f) percepción negativa de las PPL, g) las percepciones de peligrosidad (de la sobrepoblación, las pandillas, los incidentes violentos entre PPL y ciertos lugares como máxima seguridad), h) la experiencia de eventos impredecibles y traumatizantes, e i) el aislamiento de la familia (Enache, 2013; Senol, Durak, \& Gencoz, 2006).

En cuanto a las diferencias entre hombres y mujeres, Husain, Sajjad y Rehman (2014) encontraron que las policías tienden a experimentar mayores niveles de estrés, depresión y ansiedad que los hombres. Aunque el estudio se realizó con oficiales de policía, la policía tiene tareas similares a las de los oficiales penitenciarios, por lo que los resultados de dicho estudio pueden ser extrapolados. Algunos de los factores estresantes que afectan a las mujeres incluyen:

el sexismo, las actitudes negativas y las respuestas de desaprobación, la falta de modelos y tutoría, las demandas de demostrarse a sí mismas y la falta de aceptación por parte de la subcultura policial e incluso de sus propios amigos y familias. (McCarty, Zhao, \& Garland, 2007, p.673)

Sobre las mujeres, que trabajan en instituciones penitenciarias, suelen haber las siguientes quejas: los oficiales correccionales piensan que las mujeres son demasiado débiles físicamente para protegerse en enfrentamientos con PPL y a las PPL les desagrada que las mujeres les recuerden sus privaciones sexuales (Tewksbury \& Collins, 2006). A pesar de que las oficiales reportan ser víctimas de acoso en el lugar de trabajo, porque los hombres esperan que sean duras, las oficiales tienden a estar más satisfechas con su trabajo, porque están más intrínsecamente motivadas y son más productivas que los hombres (Tewksbury \& Collins, 2006). Hasta mayo de 2015, había 576 mujeres trabajando en la Policía penitenciaria de Costa Rica (Blanco \& Mora, 2015). Por lo tanto, como las mujeres son una parte considerable de la fuerza de trabajo, es importante revelar si hay alguna diferencia entre los oficiales de sexo femenino 
y masculino cuando se trata de estrés laboral. Pocos estudios han explorado disparadores de estrés laboral en oficiales penitenciarios (Campbell \& Henderson, 2016; Finney et al., 2013; Gordon \& Baker, 2015; Misis et al., 2013; Steiner \& Wooldredge, 2015; Tenibiaje, 2013), y no se ha podido encontrar ningún estudio previo que haya examinado a oficiales en Costa Rica para ese propósito. Por lo tanto, el propósito de este estudio es establecer si una muestra de oficiales penitenciarios en Costa Rica presenta estrés laboral e identificar qué factores están relacionados con su presencia. Además, este estudio examinará si existen diferencias significativas entre hombres y mujeres en las variables analizadas.

\section{Método}

\section{Participantes}

El estudio realizado fue de tipo correlacional pues como señalan Hernández, Fernández y Baptista (2006, p.105) "este tipo de estudios tienen como propósito conocer la relación que exista entre dos o más conceptos, categoría o variables en un contexto particular". Es de corte transversal no experimental porque solo se realizó una medición en la muestra, además de que como señalan Hernández et al. (2006, p.205) “(...) no se construye ninguna situación, sino que se observan situaciones ya existentes, no provocadas intencionalmente en la investigación por quien la realiza.". El estudio contó con la autorización de la Dirección de la Policía Penitenciaria del Ministerio de Justicia y Paz. El estudio se realizó tanto en la Escuela de Capacitación Penitenciaria de Costa Rica como en diversos centros de atención institucional del Circuito de Reforma durante los meses de mayo y junio de 2016. Todos los participantes firmaron un Consentimiento Informado. Un grupo de 25 oficiales y otro de 21 oficiales que se encontraban llevando capacitaciones en la Escuela fueron contactados directamente por la investigadora y aceptaron participar del estudio. Otros 20 oficiales acordaron participar en la investigación. Dichos oficiales se encontraban en servicio durante el período de recolección de datos y fueron contactados directamente por la investigadora quien visitó tres centros. La investigadora contactó, por lo menos, a 70 oficiales en los centros visitados, aunque 50 de ellos declinaron debido a que su participación implicaba responder las 120 preguntas de los instrumentos.

Los participantes necesitaban disponer de tiempo para viajar a la Escuela, o tener tiempo durante un cambio de turno o almuerzo para participar en la recolección de datos. Por tanto, la selección fue por conveniencia. Los criterios de inclusión incluyeron tener más de seis meses trabajando como oficiales penitenciarios, tener más de 20 años de edad y estar trabajando (es decir, no estar de vacaciones o incapacitados) al momento de la recolección de datos. La razón para controlar por estos criterios era asegurarse de que el estrés laboral experimentado era debido a la exposición a condiciones de trabajo.

La muestra final consistió de 66 oficiales (53 hombres y 13 mujeres). El rango de edad de los participantes fue entre 21 años a 60 años con un promedio de 34.03 años, $D E=11.75$. Solo una persona se identificó como salvadoreña. En cuanto al estado civil, 22 personas eran casadas; 22, solteras; 16, en unión libre y cinco, divorciadas. Además, 48 personas informaron tener hijos, 14 no tienen hijos y cuatro no respondieron. Los que tienen hijos reportan un promedio de 2 hijos, $D E=1$. 22. El nivel académico de la muestra incluyó un oficial con primaria incompleta, dos con primaria completa, 32 con secundaria incompleta, 25 con secundaria completa, 5 con universitaria incompleta y uno con universitaria completa. 
FACTORES ASOCIADOS AL ESTRÉS LABORAL EN POLICÍAS PENITENCIARIOS COSTARRICENSES

El promedio de meses de trabajo fue de 124.37 meses, $D E=111.57$. Además, 33 oficiales laboran en el mismo centro desde que comenzaron a trabajar como oficiales, y 33 en diferentes centros ( 1 oficial informó haber trabajado en 9 centros diferentes). La muestra incluyó oficiales de centros de todas las provincias, pero 43 oficiales trabajan en prisiones ubicadas en Alajuela. Con respecto al horario, 58 oficiales informaron trabajar en el de siete por siete (es decir, 7 días de trabajo seguidos de 7 días libres) y 8 reportaron trabajar en el de cinco por dos (es decir, 5 días de trabajo seguidos de 2 días libres). Por último, 13 oficiales informaron que tenían otro trabajo además de ser policías penitenciarios. Sus diferentes trabajos incluyeron: agricultura, apicultura, jardinería, construcción, ganadería, mercadería y amas de casa. Se pidió a la muestra que reportara las enfermedades que padecían: 14 oficiales informaron gastritis; diez, insomnio; 6, asma; 6, hipertensión; dos, migrañas y uno, úlceras. Se pidió a los oficiales que informaran si consumían cualquier sustancia (legal o ilegal): 9 oficiales informaron haber consumido tabaco; 5, antidepresivos; 2, antihistamínicos; uno, ansiolíticos; uno, 1 sedantes y uno, antipsicóticos. Ningún participante reportó el consumo de otro tipo de sustancia.

\section{Instrumentos}

Escala de Depresión, Ansiedad y Estrés-21 (DASS-21, en su versión en español). Esta escala fue desarrollada por Lovibond and Lovibond (1995) para evaluar tres constructos: depresión, ansiedad y estrés. Es una escala de Likert que consta de 21 ítems; cada subescala se compone de siete ítems. Donde por depresión se entiende la ausencia de afecto positivo, por ansiedad la hiperactivación fisiológica, y por estrés el afecto negativo (Brown, Chorpita, Korotitsch \& Barlow, 1997). La escala fue validada con una muestra costarricense por Campos, Molina y Salazar (2014) con una muestra de 329 participantes; obtuvieron un $\alpha$ de Cronbach de .75 para la escala completa, .76 para la subescala de estrés, y .74 la subescala de ansiedad (el $\alpha$ de Cronbach para la subescala de depresión no se describió).

Escala de Estrés Laboral para Oficiales Correccionales (WSSCO por sus siglas en inglés, traducido al español). Esta escala fue desarrollada por Senol et al. (2006). La escala evalúa el estrés laboral definido como "un riesgo ocupacional en el entorno penitenciario" (Senol et al., 2006, p.158). La escala es de tipo Likert, de autoinforme, y consta de 35 ítems. Se creó utilizando una muestra de 119 participantes voluntarios y demostró una buena validez concurrente y de criterio (Senol et al., 2006). La WSSCO tiene cinco subescalas: sobrecarga de trabajo, conflicto de roles y ambigüedad de roles, inadecuaciones en las condiciones físicas de la prisión, percepción de amenazas y problemas generales. Todas las subescalas están significativamente correlacionadas con las medidas de depresión, ansiedad y desesperanza en estudios previos (Senol et al., 2006). Es importante aclarar que la WSSCO se desarrolló en Turquía, por lo que solo hay validadas versiones en inglés y turco de la escala. Para fines del presente estudio, se completó una traducción al español para utilizarla con la población costarricense. La escala fue traducida al español por Tatiana Blanco, MSc., y traducida al inglés por Paola Prada, Ph.D (investigadora del Instituto de Ciencias Forenses de Texas Tech University) para asegurar la precisión del lenguaje. Según Wang, Lee y Fetzer (2006), la traducción científica apropiada incluye los siguientes pasos: “(...) una traducción hacia adelante del idioma de origen al idioma de destino, una traducción posterior del idioma de destino al idioma de origen y luego una comparación de las dos traducciones del idioma de origen “. (p. 311). 
Cuestionario Breve de Burnout (CBB, en su versión española): Este cuestionario se utiliza para medir los antecedentes y las consecuencias del constructo de burnout, entendido como el síndrome de agotamiento emocional y cinismo que ocurre con frecuencia entre las personas que tienen que trabajar cuidando a otros seres humanos. Fue desarrollado por Moreno, Bustos, Matallana, and Miralles. (1997) y debe ser utilizado con fines de investigación. Los autores reportaron buenos índices de confiabilidad y validez, con alfas de Cronbach en un rango de $\alpha=.75$ a $\alpha=.90$ para la escala global. (Moreno et al., 1997). Tiene una estructura de tipo Likert, donde 1 significa "no hay ocasión" y 5 " la mayoría de las ocasiones".

Información demográfica. Se evaluaron variables sociodemográficas de edad, género, estado civil, número de hijos, ingreso mensual aproximado, país de origen y nivel educativo. Varias preguntas se incluyeron respecto a la presencia de hipertensión, insomnio, úlceras, asma y cirrosis. Se incluyeron varias preguntas sobre el número de meses de trabajo como policía penitenciario, la ubicación del centro donde trabajan y el tipo de horario.

\section{Análisis}

Los datos fueron analizados por medio del programa estadístico SPSS (Statistical Program for Social Sciences) en su versión 22 (IBM, 2013) y el programa R versión 3.3.2 (R Core Team, 2015). Se estimaron frecuencias y estadísticos descriptivos de las variables evaluadas, se llevaron a cabo pruebas de intercorrelaciones de Pearson, pruebas T y pruebas no paramétricas de U de Mann-Whitney con el fin de establecer relaciones entre las variables cuantitativas y cualitativas, que fueron evaluadas por medio de las diferentes escalas aplicadas.

En el caso de la U de Mann-Whitney, la magnitud del efecto fue estimada siguiendo la fórmula de Cohen (1988):

$$
E S=\frac{|z|}{\sqrt{ } n}
$$

Donde $|\mathrm{z}|$ es el valor absoluto del puntaje $\mathrm{z}$ y $\mathrm{n}$ es el número total de observaciones. De acuerdo con Cohen (1988), la magnitud del efecto comprende valores desde 0 a 1 y la convención que Cohen (1988) recomendó tomar una $r=.10$ como un tamaño del efecto pequeño, $r=.30$ tamaño del efecto mediano y $r=.50$ como un efecto grande.

Dado que en todas las escalas utilizadas no todas las preguntas fueron contestadas por los participantes, hubo una alta tasa de respuestas faltantes. Por tanto, todos los datos faltantes se trataron mediante el método de la eliminación de listas (listwise).

\section{Resultados}

La tabla 1 presenta los resultados de los análisis de confiabilidad para todas las escalas utilizadas; todas las medidas demostraron una fiabilidad aceptable. La Tabla 1 también presenta las correlaciones entre las escalas, y las medias y desviaciones estándar para hombres y mujeres en esas medidas. Todas las subescalas del DASS se correlacionaron entre sí y el estrés laboral se correlacionó con todas 
las subescalas del DASS-21: con la subescala de depresión $(r=.493, p<.01)$, la subescala de estrés $(r=$ $.380, p<.05)$, y la subescala ansiedad $(r=.432, p<.01)$. El burnout solo se correlacionó con la subescala de estrés del DASS-21 $(r=.386, p<.05)$. El único resultado teóricamente inesperado fue el hecho de que el WSSCO y la CBB no tuvieron una correlación significativa.

Tabla 1

Correlaciones, medias y desviaciones estándar de la muestra $(\mathrm{N}=53$ hombres y 13 mujeres)

\begin{tabular}{|c|c|c|c|c|c|}
\hline Variable & 1 & 2 & 3 & 4 & 5 \\
\hline $1 \mathrm{WSSCO}$ & 1 & & & & \\
\hline $2 \mathrm{CBB}$ & .129 & 1 & & & \\
\hline 3 DASS DEP & $.493 * *$ & .317 & 1 & & \\
\hline 4 DASS EST & $.380^{*}$ & $.386^{*}$ & $.857^{* *}$ & 1 & \\
\hline 5 DASS ANS & $.432 * *$ & .222 & $.712 * *$ & $.852 * *$ & 1 \\
\hline$\alpha$ de Cronbach & .970 & .844 & .893 & .846 & .806 \\
\hline \multicolumn{6}{|l|}{ Mujeres } \\
\hline M & 29.33 & 61.44 & 4.33 & 4.60 & 3.12 \\
\hline SD & 18.14 & 14.15 & 5.74 & 4.37 & 3.39 \\
\hline \multicolumn{6}{|l|}{ Hombres } \\
\hline M & 37.74 & 54.02 & 2.41 & 3.08 & 2.41 \\
\hline SD & 30.64 & 12.86 & 4.13 & 3.84 & 3.54 \\
\hline
\end{tabular}

Nota $:$ WSSCO = Escala de Estrés Laboral para Oficiales Correccionales; CBB = Cuestionario Breve de Burnout; DASS DEP $=$ Subescala de Depresión; DASS EST $=$ Subescala de Estrés; DASS ANS $=$ Subescala de Ansiedad .

$* p<.05, * * p<.01$

La tabla 2 muestra las relaciones entre las variables sociodemográficas evaluadas. Se evidenció una relación significativa entre el estrés laboral y el número de niños que los oficiales reportaron tener $(r=$ $.654, p<.01)$. Hubo también una relación entre los meses de trabajo como oficial y la depresión $(r=.349$, $p<.05)$. Se identificaron dos relaciones significativas que vale la pena mencionar: una entre los meses de trabajo como oficial y la edad $(r=.829, p<.01)$ y el número de hijos $(r=.349, p<.05)$, y uno entre meses de trabajo como oficial y la cantidad de centros diferentes en los que han trabajado $(r=.566, p<.01)$.

Los resultados presentados en la Tabla 3 comparan las respuestas entre los lugares de recolección de datos. Una diferencia significativa en el burnout fue encontrada entre los oficiales que completaron las escalas en la Escuela y los que los completaron en los centros. Donde estos últimos reportaron puntuaciones más altas en burnout $(t(14.36)=-2.21, p<.05)$. No se encontraron más relaciones significativas entre los sitios de recolección. 
Tabla 2

Intercorrelaciones de Pearson para las puntuaciones del WSSCO, CBB, DASS-21 y las variables sociodemográficas

\begin{tabular}{lccccccccc}
\hline \multicolumn{1}{c}{ Variable } & 1 & 2 & 3 & 4 & 5 & 6 & 7 & 8 & 9 \\
\hline 1 WSSCO & 1 & & & & & & & & \\
2 CBB & .129 & 1 & & & & & & \\
3 DASS DEP & $.493^{* *}$ & .129 & 1 & & & & & \\
4 DASS EST & $.380^{*}$ & $.493^{* *}$ & $.857^{* *}$ & 1 & & & & \\
5 DASS ANS & $.432^{* *}$ & $.380^{*}$ & $.712^{* *}$ & $.852^{* *}$ & 1 & & & \\
6 Edad & -.080 & .285 & -.118 & -.028 & .042 & 1 & & \\
7 Cantidad de hijos & $.644^{* *}$ & .348 & .039 & .050 & .082 & .373 & 1 & \\
8 Meses de trabajar como & .231 & .069 & $.349^{*}$ & .118 & .170 & $.829^{* *}$ & $.349^{*}$ & 1 \\
policía & & & & & & & & & \\
9 Centros diferentes & -.003 & .127 & .133 & -.163 & -.065 & .588 & .061 & $.566^{* *}$ & 1 \\
\hline
\end{tabular}

Nota: WSSCO = Escala de Estrés Laboral para Oficiales Correccionales; CBB = Cuestionario Breve de Burnout; DASS DEP $=$ Subescala de Depresión; DASS EST $=$ Subescala de Estrés; DASS ANS = Subescala de Ansiedad; Centros diferentes: cantidad de centros penitenciarios diferentes en los que ha trabajado.

$* p<.05, * * p<.01$

Tabla 3

Pruebas $t$ de medias independientes de las medidas de autorreporte según lugar donde se aplicaron las pruebas

\begin{tabular}{lccccccccc}
\hline & \multicolumn{3}{c}{ Escuela } & \multicolumn{3}{c}{ Centros } & \multicolumn{3}{c}{ Resultados } \\
\hline & $\mathrm{n}$ & Media & $\mathrm{DE}$ & $\mathrm{n}$ & Media & $\mathrm{DE}$ & $\mathrm{gl}$ & $t$ & $d$ \\
WSSCO & 30 & 34.43 & 23.94 & 12 & 43.91 & 41.73 & 13.99 & -.74 & .32 \\
DASS DEP & 41 & 1.90 & 3.25 & 14 & 5.14 & 6.38 & 15.37 & -1.82 & .40 \\
DASS EST & 43 & 3.05 & 3.47 & 14 & 4.28 & 5.16 & 55.00 & -1.02 & .54 \\
DASS ANS & 44 & 2.25 & 3.29 & 12 & 3.50 & 4.19 & 54.00 & -1.09 & .56 \\
CBB & 31 & 52.25 & 10.27 & 12 & 64.16 & 16.67 & 14.36 & $-2.31^{*}$ & -2.88 \\
\hline
\end{tabular}

Nota: $\mathrm{WSSCO}=$ Escala de Estrés Laboral para Oficiales Correccionales; CBB = Cuestionario Breve de Burnout; DASS DEP $=$ Subescala de Depresión; DASS EST = Subescala de Estrés; DASS ANS = Subescala de Ansiedad.

$* p<.05$

La tabla 4 presenta la comparación de las respuestas basadas en el género; no hubo diferencias significativas entre hombres y mujeres en ninguna de las variables medidas. Sin embargo, cabe señalar que se contó con más representación de hombres que de mujeres en la muestra actual. La tabla 5 es una 
FACTORES ASOCIADOS AL ESTRÉS LABORAL EN POLICÍAS PENITENCIARIOS COSTARRICENSES

comparación de las respuestas basadas en tener hijos; hay una diferencia significativa en los puntajes de burnout y depresión para aquellos con niños, que los que no tienen. Aquellos con niños reportaron niveles significativamente más altos de depresión $(U=155, p<.05)$ y burnout $(U=71, p<.05)$.

Tabla 4

Test U de Mann-Whitney de las medidas de autorreporte según género

\begin{tabular}{|c|c|c|c|c|c|c|}
\hline & \multicolumn{2}{|c|}{ Mujeres } & \multicolumn{2}{|c|}{ Hombres } & \multirow[b]{2}{*}{$U$} & \multirow[b]{2}{*}{$r$} \\
\hline & $\mathrm{n}$ & Suma de rangos & $\mathrm{n}$ & Suma de rangos & & \\
\hline WSSCO & 3 & 62.50 & 39 & 840.50 & 56.50 & .15 \\
\hline DASS DEP & 9 & 282.00 & 46 & 1258.00 & 177.00 & .09 \\
\hline DASS EST & 10 & 343.50 & 47 & 1309.50 & 181.50 & .15 \\
\hline DASS ANS & 8 & 255.50 & 48 & 1340.50 & 164.50 & .09 \\
\hline CBB & 9 & 253.50 & 34 & 692.50 & 97.50 & .25 \\
\hline
\end{tabular}

Nota: WSSCO = Escala de Estrés Laboral para Oficiales Correccionales; CBB = Cuestionario Breve de Burnout; DASS $\mathrm{DEP}=$ Subescala de Depresión; DASS EST $=$ Subescala de Estrés; DASS ANS $=$ Subescala de Ansiedad .

Tabla 5

Test U de Mann-Whitney de las medidas de autorreporte según tenencia de hijos

\begin{tabular}{lcccccccc}
\hline & \multicolumn{2}{c}{ Hijos } & & \multicolumn{2}{c}{ No hijos } & & \\
\cline { 2 - 3 } & $\mathrm{n}$ & Suma de rangos & & $\mathrm{n}$ & Suma de rangos & & U & $\mathrm{r}$ \\
\hline WSSCO & 29 & 578.00 & & 10 & 202.00 & 143.00 & .01 \\
DASS DEP & 38 & 1080.00 & & 13 & 246.00 & & $155.00^{*}$ & .29 \\
DASS EST & 39 & 1113.50 & & 14 & 317.50 & 212.50 & .17 \\
DASS ANS & 39 & 1077.50 & & 13 & 300.50 & & 209.50 & .13 \\
CBB & 33 & 754.00 & & 8 & 107.00 & & $71.00^{*}$ & .31 \\
\hline
\end{tabular}

Nota WSSCO = Escala de Estrés Laboral para Oficiales Correccionales; CBB = Cuestionario Breve de Burnout; DASS DEP

$=$ Subescala de Depresión; DASS EST = Subescala de Estrés; DASS ANS = Subescala de Ansiedad.

$* p<.05$

La tabla 6 presenta una comparación de las respuestas basadas en tener un segundo empleo además del de policías penitenciarios. Hubo diferencias significativas en los niveles de estrés laboral, estrés y ansiedad en las personas que tienen un segundo empleo en comparación con aquellas con solo uno. Los que presentaron un segundo empleo reportaron mayores niveles de estrés laboral ( $U=90, p<0,05)$, estrés $(U=121.50, p<.05)$ y ansiedad $(U=124.50, p<.01)$. 
Tabla 6

Test $U$ de Mann-Whitney de las medidas de autorreporte según presencia de un segundo trabajo

\begin{tabular}{lcccccccc}
\hline & \multicolumn{2}{c}{ Presencia } & & \multicolumn{3}{c}{ Ausencia } & & \\
\cline { 2 - 3 } & $\mathrm{n}$ & Suma de rangos & & $\mathrm{n}$ & Suma de rangos & & \\
\hline WSSCO & 10 & 275.00 & & 31 & 586.00 & $90.00^{*}$ & .30 \\
DASS DEP & 9 & 318.50 & & 45 & 1166.50 & 131.50 & .23 \\
DASS EST & 10 & 393.50 & & 46 & 1202.50 & $121.50^{*}$ & .31 \\
DASS ANS & 11 & 425.50 & & 44 & 1114.50 & $124.50^{* *}$ & .34 \\
CBB & 11 & 245.50 & & 31 & 657.50 & 161.50 & .04 \\
\hline
\end{tabular}

Nota: $\mathrm{WSSCO}=$ Escala de Estrés Laboral para Oficiales Correccionales; CBB = Cuestionario Breve de Burnout; DASS DEP $=$ Subescala de Depresión; DASS EST $=$ Subescala de Estrés; DASS ANS $=$ Subescala de Ansiedad .

$* \mathrm{p}<.05, * * \mathrm{p}<.01$

Finalmente, las tablas 7 y 8 presentan comparaciones de cómo las enfermedades experimentadas están relacionadas con cada una de las medidas. Las personas con asma tienen mayores puntuaciones de estrés $(U=59.50, p<.05)$ que aquellas sin dicho padecimiento. La Tabla 8 indica que las personas, que sufren de insomnio, tienen diferencias significativas en depresión $(U=84.50, p<.05)$ y estrés $(U=54$, $p<.01)$. A pesar de que se evaluaron otras condiciones físicas, no tuvieron ninguna relación significativa con las puntuaciones en el WSSCO, DASS-21 o CBB.

Tabla 7

Test U de Mann-Whitney de las medidas de autorreporte según presencia de asma

\begin{tabular}{|c|c|c|c|c|c|c|}
\hline & \multicolumn{2}{|c|}{ Ausencia } & \multicolumn{2}{|r|}{ Presencia } & \multirow[b]{2}{*}{$U$} & \multirow[b]{2}{*}{$r$} \\
\hline & $\mathrm{n}$ & Suma de rangos & $\mathrm{n}$ & Suma de rangos & & \\
\hline WSSCO & 39 & 822.50 & 3 & 80.50 & 42.50 & .12 \\
\hline DASS DEP & 50 & 1377.50 & 5 & 162.50 & 102.50 & .09 \\
\hline DASS EST & 52 & 1437.50 & 2 & 215.50 & $59.50 *$ & .27 \\
\hline DASS ANS & 51 & 1433.00 & 5 & 163.00 & 107.00 & .08 \\
\hline $\mathrm{CBB}$ & 38 & 806.00 & 5 & 140.00 & 65.00 & .17 \\
\hline
\end{tabular}

Nota $:$ WSSCO = Escala de Estrés Laboral para Oficiales Correccionales; CBB = Cuestionario Breve de Burnout; DASS DEP

$=$ Subescala de Depresión; DASS EST $=$ Subescala de Estrés; DASS ANS $=$ Subescala de Ansiedad .

$* \mathrm{p}<.05$ 
FACTORES ASOCIADOS AL ESTRÉS LABORAL EN POLICÍAS PENITENCIARIOS COSTARRICENSES

Tabla 8

Test $U$ de Mann-Whitney de las medidas de autorreporte según presencia de insomnio

\begin{tabular}{lcccccccc}
\hline & \multicolumn{2}{c}{ Ausencia } & & \multicolumn{3}{c}{ Presencia } & & \\
\cline { 2 - 3 } & $\mathrm{n}$ & Suma de rangos & & $\mathrm{n}$ & Suma de rangos & & \\
\hline WSSCO & 34 & 682.50 & & 8 & 220.50 & 87.50 & .24 \\
DASS DEP & 48 & 1260.50 & & 7 & 279.50 & & $84.50^{*}$ & .30 \\
DASS EST & 51 & 1380.00 & & 6 & 273.00 & $54.00^{* *}$ & .35 \\
DASS ANS & 48 & 1297.50 & & 8 & 298.50 & 121.50 & .23 \\
CBB & 36 & 781.00 & & 7 & 165.00 & & 115.00 & .05 \\
\hline
\end{tabular}

Nota WSSCO = Escala de Estrés Laboral para Oficiales Correccionales; CBB = Cuestionario Breve de Burnout; DASS DEP $=$ Subescala de Depresión; DASS EST $=$ Subescala de Estrés; DASS ANS = Subescala de Ansiedad .

$* p<.05, * * p<.01$

\section{Discusión}

Este estudio fue correlacional, porque su propósito fue identificar la relación entre el estrés laboral y la presencia de estrés, ansiedad, depresión, burnout y diferentes características sociodemográficas y ocupacionales de una muestra de policías penitenciarios de Costa Rica. En cuanto al análisis estadístico y los resultados, las relaciones entre las variables se situaron, principalmente, en la dirección prevista, donde estrés laboral correlaciona positivamente con las demás variables evaluadas.

Las escalas utilizadas en esta investigación obtuvieron altos índices de confiabilidad. Ello indica que pueden seguir siendo utilizadas con policías penitenciarios costarricenses para efectos de investigación. En el caso específico del WSSCO, es importante mencionar que la versión en español de la escala tiene psicometría comparable a las versiones validadas en inglés y turco. Esta muestra de oficiales no puede ser comparada con las muestras utilizadas para la validación del DASS-21, porque fue validada con personas de diferentes edades. Tampoco del CBB, porque fue validada con personas españolas y no con costarricenses, lo que es una limitación.

De las variables laborales evaluadas (por ejemplo, desempeñarse en un segundo empleo, el haberse desempeñado en centros diferentes, los meses de servicio como oficial, el ingreso mensual, la situación laboral y el horario), el tener un segundo empleo tuvo un efecto significativo en los niveles de estrés laboral y de estrés y los meses de faenas como oficial tiene relación con la depresión. Además de los meses de trabajo como oficiales, otras variables laborales de su trabajo como oficiales no parecen tener una relación con el estrés laboral, el estrés, la depresión, la ansiedad o el burnout. Tener un segundo trabajo está relacionado con el estrés laboral, pero esto no significa necesariamente que otras características de su trabajo como oficiales sean las que causan el estrés laboral.

Para los participantes que reportaron consumir algún tipo de sustancia psicotrópica, esto no se asoció significativamente con la presencia de estrés laboral, estrés, ansiedad, depresión o burnout. En particular, ningún participante reportó el uso de sustancias ilícitas, lo que no es un resultado sorprendente. Se 
considera que los costarricenses tienden a no informar sobre el uso de sustancias y responder a las evaluaciones de una manera socialmente deseable (Otarola, 2015). Las investigaciones futuras deberían considerar formas alternativas de evaluar el uso de sustancias, que permitan una respuesta más honesta o incluso considerar la posibilidad de solicitar esta información en diferentes contextos que sus lugares de trabajo.

En cuanto a la presencia de enfermedades psicofisiológicas, el asma y el insomnio fueron las únicas enfermedades con relaciones significativas con estrés, depresión y burnout. Es sorprendente que la gastritis no fuera significativa, sobre todo porque se trata de la enfermedad reportada por el mayor número de oficiales, además de que la literatura presenta la gastritis relacionada con el estrés (Montaño, Dossman, Herrera, Bromet \& Moreno, 2006). Es importante tener en cuenta que el clima de muchas de los centros en el país es muy húmedo, lo que puede influir en la presencia de enfermedades respiratorias.

Es interesante que la escala, que mide el estrés laboral (WSSCO), no tuviera una relación significativa con la escala que mide el burnout (CBB). Este resultado fue completamente inesperado, dado que ambas condiciones están relacionadas con el ambiente de trabajo y una persona que experimenta estrés laboral sería propensa a experimentar también burnout (Campbell \& Henderson, 2016). Este resultado puede deberse al pequeño tamaño de la muestra y, por lo tanto, a un bajo poder estadístico. Otra posible explicación podría deberse al hecho de que ambas escalas utilizadas para este estudio no se han utilizado anteriormente en Costa Rica, y puede ser que las escalas no capturaran los constructos como se deseara, situación que debe ser explorada en futuras investigaciones.

Dado que el WSSCO se correlacionó con la subescala de depresión de la DASS-21 de la misma manera que se informó en la literatura anterior (Senol et al., 2006), se podría argumentar que el WSSCO podría ser utilizado para evaluar la presencia de la depresión también. Tener una escala que se pueda utilizar para medir diferentes condiciones psicológicas es útil para los psicólogos policiales, especialmente para usarlos con una población que no le gusta y le aburre leer como es el caso de la muestra de estudio, así los psicólogos serían capaces de obtener mucha información mediante el uso de pocas escalas.

Es importante señalar que, aunque no se encontraron diferencias significativas en las puntuaciones en las diferentes escalas entre hombres y mujeres, futuros estudios con muestras más grandes de mujeres pueden indicar diferencias significativas. Dado que en el presente estudio no se consideraron indicadores de sexismo o subculturapolicial, se podría argumentar que la posible presencia de esos indicadores en el lugar de trabajo podría influir en la forma en que las mujeres experimentan el estrés laboral y diferenciar más la experiencia de las oficiales de oficiales masculinos. Dado que en Costa Rica la violencia de género es muy común (Organización Panamericana de la Salud, 2004), una manera de erradicarla del sistema penitenciario a nivel laboral consiste en estudiar cómo se presenta y cuáles consecuencias podrían causar para las oficiales.

Dado que toda la muestra había trabajado como policías penitenciarios por más de siete meses, es posible que el estrés laboral, el estrés, la depresión, la ansiedad y el burnout, informados por los oficiales, se deban a las condiciones de trabajo que experimentan en los centros y que han experimentado esas condiciones por tiempo suficiente para que tengan efectos sobre su salud mental y física. Esas condiciones podrían incluir la supervisión de la custodia, supervisión de la vigilancia y el mantenimiento del orden y la disciplina en los centros, y proporcionar atención 24/7 a las PPL, tal como se mencionó anteriormente. En la muestra en estudio, se encontró que los oficiales también reportan consumir tabaco y experimentar 
FACTORES ASOCIADOS AL ESTRÉS LABORAL EN POLICÍAS PENITENCIARIOS COSTARRICENSES

condiciones psicofisiológicas. El hecho de que las condiciones de trabajo estén teniendo efectos en la salud mental y física de los oficiales requiere un cambio en dichas condiciones o, por lo menos, implementar una capacitación entre los oficiales para desarrollar estrategias para afrontar los muchos aspectos de ser un policía penitenciario.

Una de las limitaciones de esta investigación fue que, durante la recolección de datos, muchos oficiales se negaron a participar porque el estudio incluía leer y responder a más de 120 preguntas incluidas en los folletos. Aquellos que participaron fueron observados o declararon tener problemas para entender y contestar. Ello les tomó más tiempo de lo esperado por la investigadora. Esto revela que, para una investigación futura con oficiales costarricenses, una metodología cualitativa que involucre grupos focales o entrevistas puede facilitar la comprensión de las preguntas de la encuesta y la recopilación de información con más facilidad y profundidad.

En conclusión, altos niveles de estrés laboral, estrés, depresión, ansiedad y burnout están afectando a los oficiales penitenciarios costarricenses. La presencia de las condiciones mencionadas anteriormente tiene consecuencias a nivel físico, que pueden ser graves si no reciben atención oportuna y necesaria. Los profesionales de la salud mental deben estudiar más a fondo el problema, entender cómo las condiciones de trabajo afectan la calidad de vida de los trabajadores y su desempeño en el trabajo y proporcionar soluciones efectivas para mejorar la calidad laboral y la calidad de vida del personal penitenciario, y las condiciones de trabajo, o atendiendo directamente las consecuencias psicológicas y psicofisiológicas, que están presentes en los oficiales.

El presente estudio sirve como investigación preliminar. Proporciona información relevante sobre la presencia de condiciones psicológicas y psicofisiológicas relacionadas con el entorno de trabajo de los centros penitenciarios. La muestra informó estrés laboral y burnout que, si se deja sin atención, podría tener graves consecuencias sobre su salud física y mental. Es importante replicar este estudio con una muestra más grande para confirmar o refutar los resultados presentados en este artículo. También, resulta esencial incluir variables de sexismo por las razones anteriormente expuestas para determinar si hay diferencias significativas en cómo los hombres y las mujeres experimentan el estrés laboral, el burnout, la depresión, la ansiedad o el estrés relacionado con su trabajo como oficiales penitenciarios.

\section{Referencias}

Blanco, T., \& Mora, G. (2015). Policía penitenciaria. Manuscrito no Publicado, Facultad de Psicología, Universidad de Iberoamérica, San José, Costa Rica.

Brown, T.A., Chorpita, B.F., Korotitsch, W., \& Barlow, D.H. (1997). Psychometric properties of the Depression, Anxiety and Stress Scales (DASS) in clinical samples. Behaviour Research and Therapy, 35(I), 79-89.

Campbell, J., \& Henderson, D.F. (2016). Occupational stress: Preventing suffering, enhancing wellbeing. InternationalJournal of Environment Research andPublic Health, 13(459), 1-11. doi:10.3390/ijerph13050459

Campos, D., Molina, A., \& Salazar, K. (2014). Escalas de ansiedad y depresión del DASS-21 de Lovibond. En Smith, V. (2014). Cuaderno metodológico 6. Compendio de Instrumentos de Medición IIP-2014. Recuperado de: http://www.researchgate.net/publication/269223147 
T. Blanco-Álvarez, M. Thoen

Chandra, N., \& Parvez, R. (2016). A review article: Impact of environmental and occupational stress on health. Paripex-Indian Journal of Research, 5(5), 1-2. doi:10.15373/22501991

Cohen, J. (1988). Statistical power analysis for the behavioral sciences (2nd ed). New York: Academic Press.

Enache, R.G. (2013). Burnout syndrome and work accidents. Procedia-Social and Behavioral Sciences 78, 170174. doi:10.1016/j.sbspro.2013.04.273

Finney, C., Stergiopoulos, E., Hensel, J., Bonito, S., \& Dewa, C. (2013). Organizational stressors associated with job stress and burnout in correctional officers: a systematic review. BMC Public Health, 13(82), 1-13. Recuperado de: $\underline{\text { http://www.biomedcentral.com/1471-2458/13/82 }}$

Gordon, J., \& Baker, T. (2015). Examining correctional officers' fear of victimization by inmates: The influence of fear facilitators and fear inhibitors. Criminal Justice Policy Review, 1, 1-26. doi:10.1177/0887403415589630

Griffin, S., \& Bernard, T. (2003). Angry aggression among police officers. Police Quarterly, 6(1), 3-21. doi: $10.1177 / 1098611102250365$

Hernández, R., Fernández, C., y Baptista, P. (2006). Metodología de la investigación. (4 Ed). México:McGraw-Hill.

Husain, W., Sajjad, R., \& Rehman, A. (2014). Depression, anxiety and stress among female and male police officers. Pakistan Journal of Clinical Psychology, 13(1), 3-14.

IBM Corporation (2013). IBM SPSS Statistics for Windows, Version 22.0. New York: IBM Corporation.

Lovibond, P. F., \& Lovibond, S. H. (1995). The structure of negative emotional states: Comparison of the Depression Anxiety Stress Scales (DASS) with the Beck Depression and Anxiety Inventories. Behaviour Research and Therapy, 33(3), 335-343.

Maslach, C., \& Jackson, S.E. (1981). The measurement of experienced burnout. Journal of Organizational Behavior, 2(2), 99 - 113.

McCarty, W.P., Zhao, J., \& Garland, B.E. (2007). Occupational stress and burnout between male and female police officers: Are there any gender differences? Policing: An International Journal of Police Strategies \& Management, 30(4), 672-691. http://dx.doi.org/10.1108/13639510710833938

Misis, M., Kim, B., Cheeseman, K., Hogan, N., \& Lambert, E. (2013). The impact of correctional officer perceptions of inmates on job stress. SAGE Open; 1, 1-13. doi:10.1177/2158244013489695

Montaño, J.I., Dossman, X., Herrera, J.A., Bromet, A., \& Moreno, C.H. (2006). Helicobacter pylori y estrés psicosocial en pacientes con gastritis crónica. Colombia Médica, 37(2), 39-44.

Moreno, B., Bustos, R., Matallana, A., \& Miralles, T. (1997). La evaluación del burnout. Problemas y alternativas. El CBB como evaluación de los elementos del proceso. Revista de Psicología del Trabajo y las Organizaciones, 13(2), 185-207.

Organizacion Mundial de la Salud. (2016). Estrés laboral es una carga para los individuos, los trabajadores y las sociedades. Recuperado de http://www.paho.org/hq/index.php?option=com_content\&view=article\&id $=11973 \% 3$ Aworkplace-stress-takes-a-toll-on-individuals-employers-and-societies\&catid $=1443 \% 3 \mathrm{Aweb}-$ bulletins\&Itemid $=135 \&$ lang $=$ es 
FACTORES ASOCIADOS AL ESTRÉS LABORAL EN POLICÍAS PENITENCIARIOS COSTARRICENSES

Organización Panamericana de la Salud. (2004). La violencia social en Costa Rica. Serie Análisis de Situación de Salud, 9, 1-56. Recuperado de http://www.bvs.sa.cr/php/situacion/violencia.pdf

Otarola, M. (2015). Prevalencia de la ansiedad y de la depresión en una muestra del personal de servicios generales de la Universidad de Costa Rica, que laboran en las secciones de transportes, seguridad y tránsito. (Manuscrito no publicado). Sede Rodrigo Facio. Universidad de Costa Rica, San José, Costa Rica.

Reglamento General de la Policía Penitenciaria. (1997). Recuperado de http://www.gaceta.go.cr/pub/2012/06/15/ COMP 15 06 2012.pdf

R Core Team. (2015). R: A language and environment for statistical computing. R Foundation for Statistical Computing, Vienna, Austria. Recuperado de: https://www.R-project.org/.

Senol, E., Durak, M., \& Gencoz, T. (2006). Development of work stress scale for correctional officers. Journal of Occupational Rehabilitation, 16(1), 157-168. doi:10.1007/s10926005-9006-z

Steiner, B., \& Wooldredge, J. (2015). Individual and environmental sources of work stress among prison officers. Criminal Justice and Behavior, 42(8), 800-818. doi:10.1177/0093854814564463

Tenibiaje, D.J. (2013). Work related stress. European Journal of Business and Social Sciences, 1(10), 73-80.

Tewksbury, R., \& Collins, S. (2006). Aggression levels among correctional officers. Reassessing sex differences. The Prison Journal; 86(3), 327-343. doi:10.1177/0032885506290853

Wang, W., Lee, H., \& Fetzer, S.J. (2006). Challenges and strategies of instrument translation. Western Journal of Nursing Research, 28(3), 310-321. doi:10.1177/0193945905284712

Recibido: 30 de noviembre de 2016 Revisión recibida: 12 de mayo de 2017

Aceptado: 18 de mayo de 2017

\section{Sobre las autoras:}

Tatiana María Blanco Álvarez es Máster con máximos honores en Psicología Forense por la Universidad de Iberoamérica y es Máster en Ciencias Forenses en Texas Tech University, en Lubbock, Texas. Se graduó de Licenciada en Psicología, con honores, por la Universidad de Costa Rica y Bachiller en Ciencias Criminológicas por la Universidad Estatal a Distancia de Costa Rica. Actualmente, es estudiante Doctoral del Programa de Análisis y Resolución de Conflictos de Nova Southeastern University en Florida, Estados Unidos.

Megan A. Thoen es Doctora en Counseling Psicológico por Texas Tech University, en Lubbock, Texas. Se graduó de Máster en Psicología en Texas Tech University y Bachiller Cum Laude en Psicología por la Universidad de Mary Washington en Virginia, Estados Unidos. Funge como investigadora y como directora de los servicios forenses de salud mental del Instituto de Ciencias Forenses de Texas Tech University. Labora como Directora de la Maestría de Ciencias Forenses y docente del Doctorado en Psicología en Texas Tech University.

Publicado en linea: 26 de junio de 2017 
